\title{
Positive solutions of some nonlocal fourth-order boundary value problem with dependence on the first order derivative
}

\author{
Fei Yang ${ }^{1, a}$, YuanJian Lin ${ }^{2, b}$ \\ ${ }^{1}$ Nanchang Institute of Science and Technology, Nanchang 330108, Jiangxi \\ ${ }^{2}$ Nanchang Institute of Science and Technology, Nanchang 330108, Jiangxi \\ afeixu126@126.com, blinyuanzhou@126.com
}

Keywords: Fourth-order boundary value problem; Fixed point theorem in a cone; Positive solution

Abstract. In this paper, By the use of a new fixed point theorem and the nonlocal BVP Green function. The existence of at least one positive solutions for the nonlocal fourth-order boundary value problem with the first order derivative

$$
\left\{\begin{array}{c}
u^{(4)}(t)+A u^{\prime \prime}(t)=\lambda f\left(\mathrm{t}, u(t), u^{\prime}(t)\right), 0<t<1 \\
u(0)=u(1)=\int_{0}^{1} p(s) u(s) d s \\
u^{\prime \prime}(0)=u^{\prime \prime}(1)=\int_{0}^{1} q(s) u^{\prime \prime}(s) d s
\end{array}\right.
$$

is considered, where $f$ is a nonnegative continuous function and $\lambda>0,0<A<\pi^{2}, p, q \in L[0,1]$, $p(s) \geq 0, q(s) \leq 0$.

\section{Introduction}

The deformation of an elastic beam in equilibrium state, whose two ends are simply supported, can be described by a fourth-order ordinary equation boundary value problem. Owing to its significance in physics, it has been studied by many authors using nonlinear alternatives of Leray-Schauder, the fixed point index theory, the Krasnosel'skii's fixed point theorem and the method of upper and lower solutions, see for example [1-4, 6-10 ] .

Recently, there has been much attention focused on the question of positive solution of fourth-order differential equation with one or two parameters. For example, Li [6] investigated the existence of positive solutions for the fourth-order boundary value problem. Ma [9] studied the existence of symmetric positive solutions of the nonlocal fourth-order boundary value problem. Bai [3] studied the existence of positive solutions of the nonlocal fourth-order boundary value problem by the use of the Krasnosel'skii's fixed point theorem. All the above works were done under the assumption that the first order derivative $u^{\prime}$ is not involved explicitly in the nonlinear term $f$.

In this paper, we are concerned with the existence of positive solutions for the fourth-order three-point boundary value problem

$$
\left\{\begin{array}{c}
u^{(4)}(t)+A u^{\prime \prime}(t)=\lambda f\left(\mathrm{t}, u(t), u^{\prime}(t)\right), 0<t<1 \\
u(0)=u(1)=\int_{0}^{1} p(s) u(s) d s \\
u^{\prime \prime}(0)=u^{\prime \prime}(1)=\int_{0}^{1} q(s) u^{\prime \prime}(s) d s
\end{array}\right.
$$

The following conditions are satisfied throughout this paper:

$\left(\mathrm{H}_{1}\right) \lambda>0,0<A<\pi^{2} ;$

$\left(\mathrm{H}_{2}\right) f:[0,1] \times[0, \infty) \times R \rightarrow[0, \infty)$ is continuous, $p, q \in L[0,1], p(s) \geq 0, q(s) \leq 0$,

$\int_{0}^{1} p(s) d s<1, \int_{0}^{1} q(s) \sin \sqrt{A} s d s+\int_{0}^{1} q(s) \sin \sqrt{A}(1-s) d s<\sin \sqrt{A}$. 


\section{The preliminary lemmas}

Suppose $Y=C[0,1]$ be the Banach space equipped with the norm $\|u\|_{0}=\max _{t \in[0,1]}|u(t)|$.

Let $\lambda_{1}, \lambda_{2}$ be the roots of the polynomial $P(\lambda)=\lambda^{2}+A \lambda$, namely, $\lambda_{1}=0, \lambda_{2}=-A$. By $\left(\mathrm{H}_{1}\right)$ it is easy to see that $-\pi^{2}<\lambda_{2}<0$.

Let $Q_{i}(t, s)(i=1,2)$ be the Green's function of the linear boundary value problem :

$\left\{\begin{array}{l}-u^{\prime \prime}(t)+\lambda_{1} u(t)=0,0<t<1 \\ u(0)=u(1)=\int_{0}^{1} p(s) u(s) d s\end{array}\right.$

Then, carefully calculation yield:

$Q_{1}(t, s)=G_{1}(t, s)+\frac{1}{1-\int_{0}^{1} p(x) d x} \int_{0}^{1} G_{1}(s, x) p(x) d x ; G_{1}(t, s)=\left\{\begin{array}{l}s(1-t), 0 \leq s \leq t \leq 1 \\ t(1-s), 0 \leq t \leq s \leq 1\end{array}\right.$

$\left\{\begin{array}{l}-u^{\prime \prime}(t)+\lambda_{2} u(t)=0,0<t<1 \\ u(0)=u(1)=\int_{0}^{1} q(s) u(s) d s\end{array}\right.$

Then, carefully calculation yield:

$$
\begin{aligned}
& Q_{2}(t, s)= G_{2}(t, s)+\frac{\sin \sqrt{A} t+\sin \sqrt{A}(1-t)}{\sin \sqrt{A}-\int_{0}^{1} \sin \sqrt{A} x q(x) d x-\int_{0}^{1} \sin \sqrt{A}(1-x) q(x) d x} \int_{0}^{1} G_{2}(s, x) q(x) d x \\
& G_{2}(t, s)=\left\{\begin{array}{l}
\frac{\sin \sqrt{A} \sin \sqrt{A}(1-t)}{\sqrt{A} \sin \sqrt{A}}, 0 \leq s \leq t \leq 1 \\
\frac{\sin \sqrt{A} t \sin \sqrt{A}(1-s)}{\sqrt{A} \sin \sqrt{A}}, 0 \leq t \leq s \leq 1
\end{array}\right.
\end{aligned}
$$

Denote1: $\omega_{1}=\frac{1}{1-\int_{0}^{1} p(x) d x}, \omega_{2}(t)=\frac{\sin \sqrt{A} t+\sin \sqrt{A}(1-t)}{\sin \sqrt{A}-\int_{0}^{1} \sin \sqrt{A} x q(x) d x-\int_{0}^{1} \sin \sqrt{A}(1-x) q(x) d x}$

Lemma 2.1: Suppose $\left(\mathrm{H}_{1}\right)\left(\mathrm{H}_{2}\right)$ hold. Then for any $y(t) \in C[0,1]$, BVP

$$
\left\{\begin{array}{l}
\mathrm{u}^{(4)}(\mathrm{t})+\mathrm{Au}^{\prime \prime}(\mathrm{t})=y(t), 0<\mathrm{t}<1 \\
u(0)=u(1)=\int_{0}^{1} p(s) u(s) d s \\
u^{\prime \prime}(0)=u^{\prime \prime}(1)=\int_{0}^{1} q(s) u^{\prime \prime}(s) d s
\end{array}\right.
$$

the unique solution

$$
u(t)=\int_{0}^{1} \int_{0}^{1} Q_{1}(t, s) Q_{2}(s, \tau) y(\tau) d \tau d s
$$

where $Q_{1}(t, s)=G_{1}(t, s)+\omega_{1} \int_{0}^{1} G_{1}(s, x) p(x) d x ; \quad Q_{2}(s, \tau)=G_{2}(s, \tau)+\omega_{2}(s) \int_{0}^{1} G_{2}(\tau, x) q(x) d x$

By (3), we get: $u^{\prime}(t)=\int_{t}^{1} \int_{0}^{1} Q_{2}(s, \tau) f_{1}\left(\tau, u(\tau), u^{\prime}(\tau)\right) d \tau d s-\int_{0}^{1} \int_{0}^{1} s Q_{2}(s, \tau) f_{1}\left(\tau, u(\tau), u^{\prime}(\tau)\right) d \tau d s$

Lemma2.2 [Bai]: Assume $\left(\mathrm{H}_{1}\right)\left(\mathrm{H}_{2}\right)$ hold. Then one has:

(i) $Q_{i}(t, s) \geq 0, \forall t, s \in[0,1], Q_{i}(t, s)>0, \forall t, s \in(0,1)$;

(ii) $G_{i}(t, s) \geq a_{i} G_{i}(t, t) G_{i}(s, s), \forall t, s \in[0,1]$;

(iii) $G_{i}(t, s) \leq b_{i} G_{i}(s, s), \forall t, s \in[0,1]$.

where $a_{1}=1, a_{2}=\sqrt{A} \sin \sqrt{A} ; b_{1}=1 ; b_{2}=\frac{1}{\sin \sqrt{A}}$.

Denote2: $\quad d_{i}=\min _{\frac{1}{4} \leq t \leq \frac{3}{4}} a_{i} G_{i}(t, t)(i=1,2)$

Lemma 2.3[Bai]: Assume $\left(\mathrm{H}_{1}\right)\left(\mathrm{H}_{2}\right)$ hold and are given as above, Then one has
(i) $\max _{0 \leq t \leq 1} \omega_{2}(t)=\omega_{2}\left(\frac{1}{2}\right)$;
(ii) $0<d_{i}<1$

Lemma 2.4: If $y(t) \in C[0,1]$ and $y(t) \geq 0$, then the unique solution $u(t)$ of then BVP(1)satisfies: 
$\min _{\frac{1}{4} \leq t \leq \frac{3}{4}} u(t) \geq d_{1}\|u\|_{0} ; \quad$ where: $\quad d_{1}=\min _{\frac{1}{4} \leq t \leq \frac{3}{4}} a_{1} G_{1}(t, t)$.

Proof: By(2)and (iii) of Lemma2.2, we get:

$$
\begin{aligned}
u(t) & \leq \int_{0}^{1} \int_{0}^{1}\left[b_{1} G_{1}(s, s)+\omega_{1} \int_{0}^{1} G_{1}(s, x) p(x) d x\right] Q_{2}(s, \tau) y(\tau) d \tau d s \\
& =\int_{0}^{1} \int_{0}^{1}\left[G_{1}(s, s)+\omega_{1} \int_{0}^{1} G_{1}(s, x) p(x) d x\right] Q_{2}(s, \tau) y(\tau) d \tau d s \\
& =\int_{0}^{1} \int_{0}^{1} Q_{1}(s, s) Q_{2}(s, \tau) d \tau d s
\end{aligned}
$$

therefore, $\|u\|_{0} \leq \int_{0}^{1} \int_{0}^{1} Q_{1}(s, s) Q_{2}(s, \tau) d \tau d s$.

By (2) and (ii) of Lemma2.2, we have:

$$
\begin{aligned}
\min _{\frac{1}{4} \leq t \leq \frac{3}{4}} u(t) & \geq \min _{\frac{1}{4} \leq t \leq \frac{3}{4}} \int_{0}^{1} \int_{0}^{1}\left[a_{1} G_{1}(t, t) G_{1}(s, s)+\omega_{1} \int_{0}^{1} G_{1}(s, x) p(x) d x\right] Q_{2}(s, \tau) y(\tau) d \tau d s \\
& \geq d_{1} \int_{0}^{1} \int_{0}^{1}\left[G_{1}(s, s)+\omega_{1} \int_{0}^{1} G_{1}(s, x) p(x) d x\right] Q_{2}(s, \tau) y(\tau) d \tau d s=d_{1} \int_{0}^{1} \int_{0}^{1} Q_{1}(s, s) Q_{2}(s, \tau) d \tau d s \geq d_{1}\|u\|_{0}
\end{aligned}
$$

So, $\min _{\frac{1}{4} \leq t \leq \frac{3}{4}} u(t) \geq d_{1}\|u\|_{0}$, The proof is completed.

Theorem 2.1[guo]: Let $r_{2}>r_{1}>0, L>0$ be constants and $\Omega_{i}=\left\{u \in X: \alpha(u)<r_{i}, \beta(u)<L\right\}, i=1,2$ two bounded open sets in $X$. Set $D_{i}=\left\{u \in X: \alpha(u)=r_{i},\right\}, i=1,2$;

Assume $T: K \rightarrow K$ is a completely continuous operator satisfying:

(A $\left.\mathrm{A}_{1}\right) \alpha(T u)<r_{1}, u \in D_{1} \cap K ; \alpha(T u)>r_{2}, u \in D_{2} \cap K ; \quad\left(\mathrm{A}_{2}\right) \beta(T u)<L, u \in K$; $\left(\mathrm{A}_{3}\right)$ there is $\exists p \in\left(\Omega_{2} \cap K\right) \backslash\{0\}$, such that $\alpha(p) \neq 0$ and $\alpha(u+\lambda p) \geq \alpha(u)$, for all $\forall u \in K, \lambda \geq 0$.

Then $T$ has at least one fixed point in $\left(\Omega_{2} \backslash \overline{\Omega_{1}}\right) \cap K$

\section{The main results}

Let $X=C^{1}[0,1]$ be the Banach space equipped with the norm $\|u\|=\max _{t \in[0,1]}|u(t)|+\max _{t \in[0,1]}\left|u^{\prime}(t)\right|$, and $K=\left\{u \in X: u \geq 0, \min _{t \in\left[\frac{1}{4}, \frac{3}{4}\right]} u(t) \geq d_{1}\|u\|_{0}\right\}$ is a cone in $X$.

Define functionals $\alpha(u)=\max _{t \in[0,1]}|u(t)|, \beta(u)=\max _{t \in[0,1]}\left|u^{\prime}(t)\right|, \forall u \in X$, then $\|u\| \leq 2 \max \{\alpha(u), \beta(u)\}$, and $\alpha(\lambda u)=|\lambda| \alpha(u), \quad \beta(\lambda u)=|\lambda| \beta(u), \forall u \in X, \lambda \in R ; \alpha(u) \leq \alpha(v), \forall u, v \in K, u \leq v$.

In the following, we denote: $\eta_{0}=\int_{0}^{1} \int_{0}^{1} Q_{1}(s, s) Q_{2}(s, \tau) d \tau d s ; \quad \eta_{1}=\int_{0}^{1} \int_{\frac{1}{4}}^{\frac{3}{4}} Q_{1}(s, s) Q_{2}(s, \tau) d \tau d s$; $\eta_{2}=\mid \int_{0}^{1}\left[G_{2}(\tau, \tau)+\omega_{2}\left(\frac{1}{2}\right) \int_{0}^{1} G_{2}(\tau, x) q(x) d x\right] d \tau$

We will suppose that there are $\exists L>b>d_{1} b>c>0$ such that $f(t, u, v)$ satisfies the following growth conditions:

(H3) $f(t, u, v)<\frac{c}{\lambda d_{1} \eta_{0}}, \forall(t, u, v) \in[0,1] \times[0, c] \times[-L, L]$

(H4) $f(t, u, v) \geq \frac{b}{\lambda \eta_{1}}, \forall(t, u, v) \in[0,1] \times\left[d_{1} b, b\right] \times[-L, L]$

$\left(\mathrm{H}_{5}\right) \quad f(t, u, v)<\frac{2 L}{3 \lambda b_{2} \eta_{2}}, \forall(t, u, v) \in[0,1] \times[0, b] \times[-L, L]$

Let $f^{*}(t, u, v)=\left\{\begin{array}{l}f(t, u, v),(t, u, v) \in[0,1] \times[0, b] \times(-\infty, \infty) \\ f(t, b, v),(t, u, v) \in[0,1] \times(b, \infty) \times(-\infty, \infty)\end{array}\right.$

and $\quad f_{1}(t, u, v)=\left\{\begin{array}{c}f^{*}(t, u, v),(t, u, v) \in[0,1] \times[0, \infty) \times[-L, L] \\ f^{*}(t, u,-L),(t, u, v) \in[0,1] \times[0, \infty) \times(-\infty,-L] \\ f^{*}(t, u, L),(t, u, v) \in[0,1] \times[0, \infty) \times[L, \infty)\end{array}\right.$

Define3:

$(T u)(t)=\lambda \int_{0}^{1} \int_{0}^{1} Q_{1}(t, s) Q_{2}(s, \tau) f_{1}\left(\tau, u(\tau), u^{\prime}(\tau)\right) d \tau d s$ 


$$
(T u)^{\prime}(t)=\lambda\left[\int_{t}^{1} \int_{0}^{1} Q_{2}(s, \tau) f_{1}\left(\tau, u(\tau), u^{\prime}(\tau)\right) d \tau d s-\int_{0}^{1} \int_{0}^{1} s Q_{2}(s, \tau) f_{1}\left(\tau, u(\tau), u^{\prime}(\tau)\right) d \tau d s\right]
$$

Lemma 3.1: Suppose $\left(\mathrm{H}_{1}\right)\left(\mathrm{H}_{2}\right)$ hold , then $T: K \rightarrow K$ is completely continuous.

Proof: For $u \in K$, by (5) and (iii) of Lemma 2.2, there is $T u \geq 0$. so

$$
\begin{aligned}
\|T u\|_{0}= & \max _{t \in[0,1]}\left|\lambda \int_{0}^{1} \int_{0}^{1} Q_{1}(t, s) Q_{2}(s, \tau) f_{1}\left(\tau, u(\tau), u^{\prime}(\tau)\right) d \tau d s\right| \\
& \leq \lambda \int_{0}^{1} \int_{0}^{1}\left[b_{1} G_{1}(s, s)+\omega_{1} \int_{0}^{1} G_{1}(s, x) p(x) d x\right] Q_{2}(s, \tau) f_{1}\left(\tau, u(\tau), u^{\prime}(\tau)\right) d \tau d s \\
& =\lambda \int_{0}^{1} \int_{0}^{1} Q_{1}(s, s) Q_{2}(s, \tau) f_{1}\left(\tau, u(\tau), u^{\prime}(\tau)\right) d \tau d s
\end{aligned}
$$

By Lemma 2.2, (ii), we have :

$$
\begin{aligned}
\min _{t \in\left[\frac{1}{4}, \frac{3}{4}\right]}(T u)(t) & =\min _{t \in\left[\frac{1}{4}, \frac{3}{4}\right]} \lambda \int_{0}^{1} \int_{0}^{1} Q_{1}(t, s) Q_{2}(s, \tau) f_{1}\left(\tau, u(\tau), u^{\prime}(\tau)\right) d \tau d s \\
& \geq \lambda \int_{0}^{1} \int_{0}^{1}\left[a_{1} G_{1}(t, t) G_{1}(s, s)+\omega_{1} \int_{0}^{1} G_{1}(s, x) p(x) d x\right] Q_{2}(s, \tau) f_{1}\left(\tau, u(\tau), u^{\prime}(\tau)\right) d \tau d s \\
& \geq d_{1} \lambda \int_{0}^{1} \int_{0}^{1} Q_{1}(s, s) Q_{2}(s, \tau) f_{1}\left(\tau, u(\tau), u^{\prime}(\tau)\right) d \tau d s=d_{1}\|T u\|_{0}
\end{aligned}
$$

therefore, we get $T(K) \subset K$.

So we can get $T(K) \subset K$.Let $B \subset K$ is bounded, it is clear that $T(B)$ is bounded. Using $f_{1}, G_{1}(t, s), G_{2}(t, s)$ is continuous, we show that $T(B)$ is equicontinuous. By the Arzela-Ascoli theorem, a standard proof yields $T: K \rightarrow K$ is completely continuous.

Theorem 3.1: Suppose condition $\left(\mathrm{H}_{1}\right)-\left(\mathrm{H}_{5}\right)$ hold, Then BVP(1)has at least one positive solution $u(t)$ satisfying: $c<\alpha(u)<b, \beta(u)<L$.

Proof: Take $\Omega_{1}=\left\{u \in X:|u(t)|<c,\left|u^{\prime}(t)\right|<L\right\}, \Omega_{2}=\left\{u \in X:|u(t)|<b,\left|u^{\prime}(t)\right|<L\right\}$ two bounded open sets in $X$ and $D_{1}=\{u \in X: \alpha(u)=c\}, D_{2}=\{u \in X: \alpha(u)=b\}$.

By Lemma3.1, $T: K \rightarrow K$ is completely continuous operator, and there is $\exists p \in\left(\Omega_{2} \cap K\right) \backslash\{0\}$, such that $\alpha(p) \neq 0$ and $\alpha(u+\lambda p) \geq \alpha(u), \forall u \in K, \lambda \geq 0 . \forall u \in D_{1} \cap K, \alpha(u)=c$.

From $\left(\mathrm{H}_{3}\right)$ we get:

$$
\begin{aligned}
\alpha(T u)= & \max _{t \in[0,1]}\left|\lambda \int_{0}^{1} \int_{0}^{1} Q_{1}(t, s) Q_{2}(s, \tau) f_{1}\left(\tau, u(\tau), u^{\prime}(\tau)\right) d \tau d s\right| \\
& <\lambda \times \frac{c}{\lambda d_{1} \eta_{0}} \max _{t \in[0,1]}\left|\int_{0}^{1} \int_{0}^{1}\left[a_{1} G_{1}(t, t) G_{1}(s, s)+\omega_{1} \int_{0}^{1} G_{1}(s, x) p(x) d x\right] Q_{2}(s, \tau) d \tau d s\right| \\
& <d_{1} \times \frac{c}{d_{1} \eta_{0}} \int_{0}^{1} \int_{0}^{1}\left[G_{1}(s, s)+\omega_{1} \int_{0}^{1} G_{1}(s, x) p(x) d x\right] Q_{2}(s, \tau) d \tau d s \\
& =\frac{c}{\eta_{0}} \int_{0}^{1} \int_{0}^{1} Q_{1}(s, s) Q_{2}(s, \tau) d \tau d s=c
\end{aligned}
$$

Where as for $\forall u \in D_{2} \cap K, \alpha(u)=b$.From Lemma 2.4, we have $u(t) \geq d_{1} \alpha(u)=d_{1} b, t \in\left[\frac{1}{4}, \frac{3}{4}\right]$.so from $\left(\mathrm{H}_{4}\right)$ we get:

$$
\begin{aligned}
& \begin{aligned}
\alpha(T u)= & \max _{t \in[0,1]}\left|\lambda \int_{0}^{1} \int_{0}^{1} Q_{1}(t, s) Q_{2}(s, \tau) f_{1}\left(\tau, u(\tau), u^{\prime}(\tau)\right) d \tau d s\right| \\
& >\lambda \times \frac{b}{\lambda \eta_{1}} \max _{t \in[0,1]}\left|\int_{0}^{1} \int_{\frac{1}{4}}^{\frac{3}{4}}\left[b_{1} G_{1}(s, s)+\omega_{1} \int_{0}^{1} G_{1}(s, x) p(x) d x\right] Q_{2}(s, \tau) d \tau d s\right| \\
& =\frac{b}{\eta_{1}} \int_{0}^{1} \int_{\frac{1}{4}}^{\frac{3}{4}} Q_{1}(s, s) Q_{2}(s, \tau) d \tau d s=b
\end{aligned} \\
& \forall u \in K, \text { From }\left(H_{5}\right) \text { we get: } \\
& \beta(T u)= \max _{t \in[0,1]}\left|\lambda \int_{t}^{1} \int_{0}^{1} Q_{2}(s, \tau) f_{1}\left(\tau, u(\tau), u^{\prime}(\tau)\right) d \tau d s-\lambda \int_{0}^{1} \int_{0}^{1} s Q_{2}(s, \tau) f_{1}\left(\tau, u(\tau), u^{\prime}(\tau)\right) d \tau d s\right| \\
&<\lambda \times \frac{2 L}{3 \lambda b_{2} \eta_{2}} \max _{t \in[0,1]}\left|\int_{0}^{1} \int_{0}^{1}(1+s) Q_{2}(s, \tau) d \tau d s\right| \\
&<\frac{2 L}{3 b_{2} \eta_{2}}\left|\int_{0}^{1} \int_{0}^{1}(1+s)\left[b_{2} G_{2}(\tau, \tau)+\max _{s \in[0,1]} \omega_{2}(s) \int_{0}^{1} G_{2}(\tau, x) q(x) d x\right] d \tau d s\right|
\end{aligned}
$$




$$
<\frac{2 L}{3 b_{2} \eta_{2}} \times \frac{3}{2} b_{2}\left|\int_{0}^{1}\left[G_{2}(\tau, \tau)+\omega_{2}\left(\frac{1}{2}\right) \int_{0}^{1} G_{2}(\tau, x) q(x) d x\right] d \tau\right|=L
$$

Theorem 2.1 implies there is $u \in\left(\Omega_{2} \backslash \bar{\Omega}_{1}\right) \cap K$, such that $u=T u$.so, $u(t)$ is a positive solution for BVP(1), satisfying : $c<\alpha(u)<b, \max _{t \in[0,1]}\left|u^{\prime}(t)\right|<L$.

Thus, Theorem 3.1is completed.

\section{References}

[1] Z.B. Bai, The method of lower and upper solution for a bending of an elastic beam equation, $J$. Math. Anal. Appl. 248 (2000) 195-202.

[2] Z.B. Bai, The method of lower and upper solutions for some fourth-order boundary value problems, Nonlinear Anal. 67 (2007) 1704-1709.

[3] Z.B. Bai, Positive solutions of some nonlocal fourth-order boundary value problem, Applied Mathematics and Computation 215 (2010) 4191-4197

[4] G.Q. Chai, Existence of positive solutions for fourth-order boundary value problem with variable parameters, Nonlinear Anal. 66 (2007) 870-880.

[5] D. Guo, V. Lakshmikantham, Nonlinear Problems in Abstract Cones, Academic Press, New york, 1988.

[6] Y.X. Li, Positive solutions of fourth-order boundary value problems with two parameters, $J$. Math. Anal. Appl. 281 (2003) 477-484.

[7] R. Ma, H.Wang, on the existence of positive solutions of fourth-order ordinary differential equations, Appl.Anal. 59 (1995) 225-231.

[8] Yanping Guo, Positive solutions for three-point boundary value problems with dependence on the first order derivatives, Journal of Mathematical Analysis and Applications 290 (2004) 291-301.

[9] H.L. Ma, Symmetric positive solutions for nonlocal boundary value problems of fourth-order, Nonlinear Anal. 68 (2008) 645-651.

[10] Q.L. Yao, Local existence of multiple positive solutions to a singular cantilever beam equation, J. Math. Anal. Appl. 363 (2010) 138-154.

[11] R.Y. Ma, Existence of positive solutions of a fourth-order boundary value problem, Appl. Math. Comput. 168 (2005) 1219-1231.

[12] J.F. Zhao, W.G. Ge, Positive solutions for a higher-order four-point boundary value problem with a p-Laplacian, Comput. Math. Appl. 58 (2009) 1103-1112. 